\title{
SINTOMATOLOGÍA ASOCIADA A NEUROCISTICERCOSIS EN PERSONAS DEL ÁREA URBANA Y PERIURBANA DEL MUNICIPIO DE COYAIMA DEL DEPARTAMENTO DEL TOLIMA Y POSIBLES VARIABLES ASOCIADAS
}

\author{
JULIO CÉSAR GIRALDOํ, DIANA MARCELA CHALA², LUIS REINEL VÁSQUEZ, \\ TOMAS ÓMAR ZAMORA4 , JUAN CARLOS CASAS 5
}

${ }^{1}$ Msc. Esp. Docente Investigador, líder Grupo de Investigación en Parasitología y Microbiología Tropical (GIPAMT). Facultad de Ingeniería, Administración y Ciencias Básicas. Programa de Biología. Universidad INCCA de Colombia. Bogotá, Colombia ${ }^{2}$ Bióloga. Universidad INCCA de Colombia. Bogotá, Colombia

${ }^{3}$ Msc. Esp. Docente Investigador, líder Grupo Centro de Estudios en Microbiología y Parasitología (CEMPA). Facultad de Ciencias de la Salud, Departamento de Medicina Interna. Universidad del Cauca. Popayán, Cauca, Colombia

${ }^{4}$ MD. Neurólogo. MD Internista, Centro de Estudios en Microbiología y Parasitología (CEMPA), Facultad de Ciencias de la Salud. Departamento de Medicina Interna. Universidad del Cauca. Popayán, Cauca, Colombia. MD Neurólogo y MD Internista, Hospital Universitario San José, Popayán, Cauca, Colombia

${ }^{5}$ MD Neurólogo Liga Colombiana contra la Epilepsia, capítulo Cauca (LCEC) Clínica La Estancia, Popayán, Cauca, Colombia ${ }^{1}$ Correspondencia: Julio Cesar Giraldo. jcesargiraldo@gmail.com Recibido: 3 Febrero 2016 Aceptado: 6 Julio 2016

\section{RESUMEN}

Introducción: El complejo teniasis/cisticercosis es un problema de salud pública a nivel mundial. En Colombia estudios epidemiológicos evidencian la presencia de ésta parasitosis en humanos ocasionando Neurocisticercosis (NCC).

Objetivo: Determinar la sintomatología asociada a Neurocisticercosis en personas del área urbana y periurbana del municipio de Coyaima del departamento del Tolima y posibles variables asociadas.

Materiales y Métodos: Se realizó un estudio descriptivo de corte transversal en los habitantes del área urbana y periurbana del municipio de Coyaima en el departamento del Tolima, a quienes se les socializó un consentimiento informado. A las 159 personas que aceptaron participar voluntariamente, se les diligenció una encuesta epidemiológica estructurada, toma de $5 \mathrm{ml}$ de sangre periférica y simultáneamente por punción capilar sangre colectada en círculos de papel filtro de $4 \mathrm{~mm}$ diámetro; de igual manera se solicitó una muestra coprológica que se fijó con reactivo de MIF al $5 \%$ y se procesó con la técnica de concentración de formol-éter y finalmente almacenadas a $4^{\circ} \mathrm{C}$. Para la evaluación serológica, se empleó la técnica de ELISA bajo los siguientes parámetros: antígeno, fracción de 53kDa del 
metacéstodo de Taenia solium.; en concentración de $0,4 \mu \mathrm{g} / \mathrm{ml}$, dilución de suero y sangre total tomada con papel de filtro1/1, suero total 1/100 y conjugado 1/2500. El análisis estadístico incluyo tablas de contingencia de $2 \times 2$, cálculo de frecuencias y ji cuadrado.

Resultados: De los 159 participante, el 17\% (27/159) fueron diagnosticados seropositivos y de estos, el 67\%(18/27) presentó alguna sintomatología de posible asociación con NCC. Los síntomas de mayor frecuencia en la población estudiada fueron: cefalea, 19,5\%; disminución de capacidad visual, 5,7\%; crisis convulsiva de aparición tardía, 4,4\%; accidentes cerebrovasculares y alteración de conciencia $1,3 \%$ y $0,6 \%$; respectivamente. Se confirmaron cuatro personas con NCC, del grupo de seropositivos (4/27) tres de género femenino y uno masculino, por técnicas de imagenología como Tomografía Axial Computarizada-TAC o Resonancia Magnética-RM. Las sintomatologías de mayor relevancia para estos pacientes fueron crisis convulsiva de aparición tardía y cefalea. La prevalencia general de parasitismo intestinal en la población participante fue del $43.3 \%$, identificándose en el $2 \%(3 / 159)$ de los valorados, huevos de Taenia spp., y de estos una persona a su vez seropositiva, con sintomatología (crisis convulsiva de aparición tardía y cefalea) compatible con NCC y confirmación posterior por TAC, sugiriéndose una parasitosis mixta por el estadio adulto y el metacéstodo de T. solium.; en el paciente.

Conclusiones: El 17\%(27/159) de la población estudiada fue seropositiva, se confirmaron cuatro personas por imagenología con NCC, cuyos síntomas de mayor relevancia fueron crisis convulsiva de aparición tardía y cefalea. El género femenino es proclive a sufrir la parasitosis. Se halló presencia de huevos de Taenia. spp.; en el $2 \%$ de la población participante en el estudio. La variable fuentes de agua para consumo presento significancia estadística $\mathrm{p} \leq 0,05$.

Palabras claves: Neurocisticercosis, Taenia solium.; ELISA, Papel filtro, Variables asociadas, Sintomatología asociada. 


\title{
SYMPTOMS ASSOCIATED WITH NEUROCYSTICERCOSIS IN PEOPLE IN THE URBAN ND PERI-URBAN OF THE MUNICIPALITY OF COYAIMA THE DEPARTMENT OF TOLIMA AND POSSIBLE ASSOCIATED VARIABLES
}

\begin{abstract}
Introduction: The complex teniasis/cysticercosis is a worldwide public health problem. Colombia epidemiological studies shows the presence of parasites in humans resulting in neurocysticercosis (NCC).

Objective: To determine the symptoms associated with neurocysticercosis in people in the urban and peri-urban area of the town of Coyaima in the Department of Tolima and possible associated variables.

Materials and methods: a descriptive cross-sectional study was performed in the inhabitants of the urban and peri-urban area of the town of Coyaima in the Department of Tolima, who socialized informed consent. The 159 people that they agreed to participate voluntarily, is filled them a structured epidemiological survey, $5 \mathrm{ml}$ of blood making peripheral and simultaneously by finger stick blood collected in $4 \mathrm{~mm}$ diameter filter paper circles; Similarly be asked a coprological sample that was set with MIF reagent to $5 \%$ and was processed with the concentration of formol-eter technique and finally stored at $4{ }^{\circ} \mathrm{C}$. Serologic evaluation, used the ELISA technique under the following parameters: Antigen fraction of $53 \mathrm{kDa}$ of the metacestodo of Taenia solium.; concentration of $0,4 \mu \mathrm{g} / \mathrm{ml}$, dilution of serum and whole blood taken with filter $1 / 1$, total serum conjugated 1/2500 and 1/100 paper. Statistical analysis included calculation of frequencies and Chi square $2 \times 2$ contingency tables.

Results: Of 159 participants, 17\%(27/159) were diagnosed positive, and of these, 67\%(18/27) presented any symptoms of possible partnership with NCC. Symptoms of greater frequency in the population studied were: headache, 19.5\%; decrease of visual acuity, 5.7\%; late-onset seizure, $4.4 \%$; accidents cerebrovascular and alteration of consciousness $1.3 \%$ and $0.6 \%$; respectively. Confirmed four people with NCC, of the positive Group(4/27) three female and one male, by techniques such as tomography Axial-TAC or magnetic resonance-MRI Imaging. The symptomatology of greatest relevance for these patients was seizure of late-onset and headache. The overall prevalence of intestinal parasitism in the participant population was $43.3 \%$, identified in the $2 \%(3 / 159)$ rated, eggs of Taenia spp.; and of these a person positive turn, with symptoms (seizure of late-onset and headache) compatible with NCC and subsequent confirmation by TAC, suggesting a mixed parasitic infections by the adult stage and the metacestodo of $T$. solium.; in the patient.

Conclusions: $17 \%(27 / 159)$ of the studied population was positive, four persons were confirmed by imaging with NCC, whose symptoms of greater importance were seizure of late-onset and headache. The feminine gender is prone to suffer parasitosis. We found the presence of eggs of Taenia. spp.; in $2 \%$ of the population participating in the study. The variable sources of water for consumption presented statistical significance $p \leq 0.05$.
\end{abstract}

Key words: Neurocysticercosis, Taenia solium.; ELISA, Paper filter, Variable associated, Associated symptoms. 


\title{
SINTOMAS ASSOCIADOS A NEUROCISTICERCOSE EM PESSOAS DA ZONA URBANA E PERI URBANA DO MUNICÍPIO COYAIMA DO DEPARTAMENTO DE TOLIMA E VARIÁVEIS ASSOCIADAS POSSÍVEIS
}

\begin{abstract}
RESUMO
Introdução: O complexo teníase / cisticercose é um problema de saúde pública mundial. Estudos epidemiológicos na Colômbia mostram a presença de parasitasem seres humanos resultando em neurocisticercose (NCC).

Objetivo: Determinar os sintomas associados à neurocisticercose em pessoas na área urbana e peri-urbana da cidade de Coyaima no Departamento do Tolima e possiveis variáveis associadas.

Materiais e métodos: Um estudo descritivo transversal foi realizado nos habitantes da área urbana e peri-urbana da cidade de Coyaima, no Departamento do Tolima, que socializou o consentimento informado. As 159 pessoas que concordaram em participar voluntariamente, e preenchido um inquérito epidemiológico estruturado, 5 ml de sangue fazendo periférico e simultaneamente por sangue de vara de dedo coletado em círculos de papel de filtro de $4 \mathrm{~mm}$ de diâmetro; Do mesmo modo, ser-lhe-á solicitada uma amostra coprológica que foi fixada com reagente MIF a 5\% e processada com a concentração de formol-eter e finalmente armazenada a $4{ }^{\circ} \mathrm{C}$. A avaliação sorológica utilizou a técnica ELISA sob os seguintes parâmetros: Fração de antígeno de 53kDa do metacesto de Taenia solium, concentração de $0,4 \mu \mathrm{g} / \mathrm{ml}$, diluição de soro e sangue total coletado com filtro1/1, soro total conjugado com $1 / 2500$ e 1/100 de papel. A análise estatística incluiu o cálculo de frequências e tabelas de contingência Chi 2 × 2 .
\end{abstract}

Resultados: Dos 159 participantes, 17\% (27/159) foram diagnosticados positivos, e destes, 67\% (18/27) apresentaram qualquer sintoma de possivel parceria com a NCC. Os sintomas de maior freqüência na população estudada foram dor de cabeça, 19,5\%; Diminuição da acuidade visual, 5,7\%; Ataque tardio, 4,4\%; Acidentes vasculares cerebrais e alteração da consciência 1,3\% e 0,6\%; respectivamente. Confirmou quatro pessoas com NCC, do grupo positivo (4/27) três do sexo feminino e um do sexo masculino, por técnicas como a tomografia Axial-TAC ou ressonância magnética-Imaging RM. A sintomatologia de maior relevância para estes pacientes foi a convulsão de início tardio e dor de cabeça. A prevalência total de parasitismo intestinal na população participante foi de 43,3\%, identificados nos 2\% (3/159) avaliado, ovos de Taenia spp., e destes, por sua vez, uma pessoa positiva, com sintomas (apreensão de início tardio e dor de cabeça) compativel com NCC e subsequente confirmação por TAC, sugerindo uma mistos infecções parasitárias por a etapa de adultos e o metacestodo de T. solium., no paciente.

Conclusões: 17\% (27/159) da população estudada foi positiva, quatro pessoas foram confirmadas por imagem com NCC, cujos sintomas de maior importância foram apreensão de início tardio e cefaléia. O sexo feminino é propenso a sofrer parasitose. Encontramos a presença de ovos de Taenia spp., em $2 \%$ da população participante do estudo. As fontes variáveis de água para consumo apresentaram significância estatística $p \leq 0,05$.

Palavras-chave: Neurocisticercose, Taenia solium spp., ELISA, Filtro de papel, Variável associada, Sintomas associados. 


\section{Introducción}

La Cisticercosis humana es una parasitosis producida por el céstodo Taenia solium.; en su forma larval, esté puede llegar a difundirse en todo el sistema nervioso central (SNC) lo cual despliega un conjunto de sintomatologías; siendo la Neurocisticercosis (NCC) la enfermedad más relevante en el aspecto clínico (1), puede causar convulsiones, alteraciones en la conducta, hidrocefalia obstructiva y otros signos neurológicos (2), estas sintomatologías van estrechamente relacionadas con la respuesta humoral del huésped, dependiendo del curso de la infección, es decir activa o manifiesta, hay una reacción inmunológica mediada por anticuerpos cuando el estadio larval infectivo se encuentra vivo, generando mecanismos de evasión del sistema inmunológico, como son: la fijación de anticuerpos del hospedero en su membrana vesicular y así pasar desapercibido, como también la ubicación en lugares del huésped inmunológicamente privilegiados.

Los anticuerpos generados en defensa de esta parasitosis son más abundantes cuando el estadio está vivo que cuando este muere y calcifica, debido a la presencia de reacciones metabólicas, genera una serie de biomoléculas como la alfa ( $\alpha$ ) estatina inhibidora de la serina que a su vez no permite el desarrollo de la respuesta inflamatoria en el organismo invadido. Caso contrario ocurre cuando la larva muere y se transforma en un cúmulo de calcio, cesa la inhibición y se da una respuesta de ataque en la que el sistema de defensa, genera una mayor reacción frente a la infección parasitaria y en algunos casos acrecentándose la sintomatología y patología (3).

El ciclo de vida de T. solium.; en su etapa adulta se localiza adherida la pared del intestino delgado del humano y a partir de ésta, se expulsan proglótides grávidas las cuales contienen huevos, que pueden oscilar en número de 40 y 60.000 por cada una, las que son fuente de infección para los porcinos por su actividad coprofágica, los embriones hexacanto atraviesan la pared intestinal y son distribuidos por el sistema circulatorio al musculo esquelético, cardiaco y sistema nervioso central, donde se origina el estadio vesicular o metacéstodo, siendo éste, el que da curso infectivo para originar la fase adulta del parasito en el huésped definitivo que es el hombre; mediante la ingestión de carne con malos procesos de cocción y cisticercos vivos. El desarrollo del ciclo de vida, para esta parasitosis desde estadio oval hasta la fase adulta se puede lograr en un periodo de diez a doce semanas (4).
La NCC es una enfermedad parasitaria incapacitante, en Colombia como en otras zonas endémicas de África, Asia y Latino-América, es considerada un problema de salud pública y una de las principales causas es la epilepsia adquirida, situación que se ve favorecida en zonas con deficiencia en ingeniería sanitaria, saneamiento ambiental, educación y crianza no tecnificada de cerdos $(1,5,6)$.

En 1993, la International Task Force for Disease Erradication, declara que la cisticercosis es una enfermedad zoonotica potencialmente erradicable según U.S. Department Of Health And Human Services (7).

El cálculo para el comportamiento de la enfermedad a nivel mundial estima que aproximadamente 2.500.000 individuos son portadores de T. solium.; y alrededor de 20.000.000 de personas están infectadas por el parasito en su estado larval. Por tanto, se relaciona que por cada teniásico puede haber ocho individuos con cisticercosis con o sin sintomatología asociable (8).

La dispersión de la enfermedad es amplia en Colombia, como lo indica el estudio realizado en 23 departamentos para el periodo 2008-2010, reportando una seroprevalencia general de $8.55 \%$ a nivel nacional, el departamento de mayor frecuencia, Vaupés (40.19\%) y Caldas el de menor (0.53\%). Algunos factores asociados a la cisticercosis hallados en el estudio fueron: nivel educativo no mayor a la secundaria, estrato socioeconómico bajo, ocupación, predilección gastronómica en el consumo de carne de cerdo y ocasionalmente o nunca lavado de verduras con agua potable (1).

El diagnóstico de NCC en Colombia es limitado debido al costo que tienen metodologías serológicas como ELISA y Western Blot, e Imagenología como Tomografía Axial Computarizada -TAC y Resonancia Magnética - RMC. Algunos autores han reportado que se puede dar el caso en que pacientes con NCC confirmada, presenten títulos bajos de anticuerpos u ocasionalmente ninguno, en suero o en líquido cefalorraquídeo (LCR) $(1,6)$.

La fracción de $53 \mathrm{kDa}$ del metacéstodo de T. solium.; presenta alta especificidad para la fase larval o vesicular, sin reporte de reacciones cruzadas con otros helmintos, tales como adultos de T. solium.; T. saginata.; Echinococcus spp.; Ascaris lumbricoides.; Enterobius vermicularis.; Strongyloides stercoralis.; y Uncinarias (9).

La técnica de recolección de sangre total en papel de filtro $(\mathrm{PF})$ ha reportado ser útil desde la década de los 
70s; siendo empleada en varias partes del mundo para el diagnóstico de enfermedades infecciosas en animales y humanas, demostrando en las diferentes técnicas de serodiagnóstico, valores elevados de sensibilidad, especificidad, predictivos positivo y negativo; $y$ bondades como facilidad de uso en campo, comodidad para el transporte de la muestra, poca ocupación de espacio en la conservación, entre otras, como se ha sido demostrado en estudios en los cuales se ha empleado ésta, como lo reportado para Leishmaniosis, enfermedad de Chagas y toxoplasmosis $(10,11)$.

Los resultados de esta investigación, se socializaron con la comunidad participante para la búsqueda de alternativas viables que permitan contribuir en la posible erradicación de la enfermedad en la población Coyaimuna como también en el control de la parasitosis, desde el cambio de conductas higiénico-sanitarias inapropiadas, que contribuyen al establecimiento de la misma.

\section{Metodología}

\section{Área y población de estudio}

Se realizó un estudio descriptivo de corte transversal, con toma única de muestra en los habitantes del área urbana y periurbana del municipio de Coyaima en el departamento del Tolima, que posee un área territorial de $664.33 \mathrm{~km}^{2}$, los cuales $658,13 \mathrm{~km}^{2}$ corresponden al sector rural (99\%) y $6,20 \mathrm{~km}^{2}$ al sector urbano (1\%), se halla a 392MSNM, con piso térmico cálido y temperatura promedio de $27^{\circ} \mathrm{C}$. En la prestación de servicio de salud, el municipio cuenta con un hospital de primer nivel, único centro de atención para la población de la región.

\section{Recolección de los datos}

Se realizó un muestreo uniforme en el área de estudio, de Junio a Diciembre del año 2013, con visita domiciliaria en el que se impartió charlas informativas y suministro de material didáctico del ciclo epidemiológico del complejo teniosis/cisticercosis y factores asociados. Se procedió a la firma de un consentimiento informado y la aplicación de una encuesta estructurada, la cual incluyó información sociodemográfica y ecoepidemiológica como edad, servicio de salud, nivel educativo, variables y sintomatología vinculadas con especial énfasis a la NCC.

\section{Recolección de Muestras Serológicas Humanas}

La toma de muestras se realizó en dos fases a 159 personas del área urbana y periurbana del municipio, incluyendo siete pacientes que por información obtenida de las historias clínicas proporcionadas por el cuerpo médico del hospital, asistieron a consulta entre el periodo de Junio de 2010 a Junio de 2013 por presentar crisis convulsiva de aparición tardía, sintomatología compatible con NCC.

\section{Toma de muestra serológica}

Extracción de $5 \mathrm{ml}$ de sangre por punción venosa del brazo, que se centrifugó a 2500rpm para obtención del suero, que se alicuotó y refrigeró a $4^{\circ} \mathrm{C}$; seguidamente punción digital del dedo índice de la mano izquierda con lanceta estéril e impregnación en círculos de papel de filtro de $4 \mathrm{~mm}$ de diámetro; Wattman No 1 que se dispensaron en tubos Eppendorf y se refrigeraron a $4^{\circ} \mathrm{C}$.

\section{Toma de Muestra Coprológica}

A todos los participantes se les suministró un recipiente codificado para la recolección de una muestra de materia fecal. En el momento de la recepción de ésta, se le adicionó $30 \mathrm{ml}$ de reactivo de MIF (Mertiolate, Yodo, Formol) al 5\%, mezclándose hasta su homogenización; y se preservó a $4^{\circ} \mathrm{C}$. Transportadas las muestras al laboratorio, se procesaron empleando de la técnica de concentración de formol-éter, para el diagnóstico se realizó examen coproparasitológico por triplicado y doble ciego.

\section{Prueba serológica}

La prueba implementada fue un ELISA de tipo indirecto, utilizando la fracción $53 \mathrm{kDa}$ como antígeno de cisticerco de T. solium.; proveniente del estadio vesicular del parasito, el cual se fijó en placas de policarbonato. A cada suero problema se le realizó en una dilución de $1 / 100$. Posteriormente se adicionó una anti gammaglobulina humana Anti-IgG unida a la enzima peroxidasa. Este procedimiento fue realizado con el total de los sueros. Las muestras fueron consideradas como positivas cuando estas superaron el valor del punto de corte de 0.535(9).

Para la valoración de las muestras recolectadas en círculos de papel filtro de $4 \mathrm{~mm}$ de diámetro Wattman $\mathrm{N}^{\circ} 1$; se adecuo a la prueba ELISA previamente descri- 
ta, diluyendo por agitación el contenido de sangre total de cada círculo de papel en $1000 \mu l$ de solución salina fisiológica, de esta se tomaron $100 \mu \mathrm{l}$ correspondiendo a una dilución de $1 / 1$.

\section{Análisis estadístico}

Se construyó una base de datos con las variables evaluadas en el estudio: sociodemográficas y clínicas, entre otras; con el empleo del paquete estadístico $\mathrm{SPSS}^{\mathrm{v} 20}$ se realizó un análisis bivariado descriptivo.

\section{Resultados}

Caracterización general de la comunidad del municipio de Coyaima del Departamento del Tolima.

El estudio contó con la participación voluntaria de 159 personas, la distribución por genero correspondió $68 \%$ al femenino y $32 \%$ al masculino, hallándose en 27 participantes anticuerpos contra el metacéstodo de T. solium.; arrojando un $17 \%$ de seropositividad para la población estudiada. De los casos positivos, 11 personas (40.7\%) fueron del género masculino y 16 (59.3\%) del femenino, cabe destacar que el 67\%(18/27) de los seropositivos presentaron al menos un síntoma asociable a NCC.

La población evaluada se distribuyó en tres grupos etáreos: el primero conformado de 1-23 años con 29 personas (18\%), del cual siete presentaron anticuerpos anticisticerco, el segundo grupo de 24-35; compuesto por 51 participantes (32\%), de los cuales ocho fueron positivos y finalmente el tercer grupo conformado por los mayores de 36 años, con 79 personas, siendo este el mayoritario con un $50 \%$, de los cuales 12 resultaron positivos.

La distribución por procedencia fue: zona urbana 105 (66\%) y periurbana 54 (34\%) con 18 y nueve casos de seropositivos respectivamente.

En cuanto a ocupación de la población, se identificó que el 44,6\% no estaba empleada en el momento del estudio; el 55,4\% complementario, se distribuyó de la siguiente manera: trabajador independiente $28,3 \%$, empleados públicos y privados $8.8 \%$, estudiantes $17,6 \%$ y pensionados $0,63 \%$. El grupo laboral independientes presentó el 33,3\% (9/27) seropositividad, seguido de estudiantes y desempleados 59,3\%(16/27), con ocho personas cada uno y los grupos restantes, empleados y pensionados $7,4 \%(2 / 27)$; con un seropositivo respectivamente.

Otra variable que incluyó el estudio fue, el régimen de salud con la siguiente distribución: la Aseguradora de Riesgos de Salud (ARS) dos personas (1,2\%); Entidad Promotora de Salud (EPS) con 133 contribuyentes (83,6\%); Sistema de Identificación y Clasificación de Potenciales Beneficiarios (SISBEN) con 21 individuos $13,2 \%$, desplazado solo un caso $0,63 \%$ y sin ningún régimen de salud dos participantes 1,2\%. De esta variable, se presentó el mayor número de seroprevalentes para la EPS con 96,3\%(26/27), seguido por el SISBEN con $3,7 \%(1 / 27)$. El grupo conformado por desplazados y los que no contaron con algún tipo de régimen en salud, fue seronegativo. La variable régimen de salud fue estadísticamente significativa en los análisis de los resultados para el caso de prueba de ELISA como para la sintomatología asociada a NCC con $\mathrm{p} \leq 0,05$. El grado de escolaridad de los participantes se distribuyó de la siguiente manera, iletrados 12\% (19/159), educación primaria $46,5 \%$ (74/159), secundaria $33,3 \%(53 / 159)$, nivel tecnológico 6,3\% (10/159) y de nivel de educación superior $2 \%(3 / 159)$. En la tabla 1 se presentas los valores porcentuales de las variables demográficas y clínicas.

Un aspecto relevante en el estudio fue la relación epidemiológica y el desarrollo del ciclo de vida del parásito, con manifestaciones clínicas y sintomatología con posible asociación a NCC. Lo manifestado por los participantes en cuanto haber tenido contacto con porcinos directamente en procesos de crianza fue del $76,7 \%(122 / 159)$ y de ellos el $13 \%$ se diagnosticaron seropositivos. En el momento del levantamiento de la información, el 23,2\% (37/159) de los encuestados asintieron estar practicando la actividad de crianza de animales en condiciones no tecnificadas, de este grupo, el $4 \%$ presentaron anticuerpos antimetacéstodo.

Otra variable evaluada fue las fuentes de agua que abastecen a los habitantes de la población, de acuerdo con ella 91,2\% (145/159) manifestó no poseer en sus viviendas dotación de acueducto $\mathrm{y} / \mathrm{o}$ alcantarillado, se observó que la totalidad de casos seropositivos presentaron esta condición higiénico-sanitaria, (27/27) $100 \%$. La variable suministro de agua y dotación de alcantarillado presentó significancia estadística con valor de $\mathrm{p} \leq 0,05$, dando lugar a sugerir que estas pueden estar relacionadas con la presencia de la parasitosis en la población. En tabla 2 se relacionan los 
valores porcentuales de tipo de crianza de los cerdos, satisfacción de condiciones básicas y procedencia de los participantes.

\section{Sintomatología Asociada a NCC}

El 42\%(67/159) de la población incluida en el estudio manifestó presentar al menos un síntoma asociable a NCC.

La sintomatología se discriminó de la siguiente manera:

Cefalea

El 19,5\%(31/159) de los participantes manifestaron presentar esta afección, al confrontar este resultado con lo reportado por otros autores, hacen alusión que este síntoma es prevalente en los pacientes con NCC confirmada; más no es concluyente de la enfermedad. En el estudio, 14,8\%(4/27) de los seropositivos se les confirmo el diagnóstico de NCC por imagenología con RM y/o TAC, los cuales el 75\%(3/4) presentaron como síntoma en común, cuadros de cefalea frecuentes.

\section{Disminución de capacidad visual}

El 5,7\%(9/159) de los participantes, manifestaron presentar disminución en su capacidad visual; hallándose significancia estadística entre esta variable y la valoración serológica con valor de $\mathrm{p} \leq 0,05$.

Crisis convulsiva de aparición tardía.

El 4,4\% (7/159) asistieron a consulta médica entre el periodo de Junio de 2010 a Junio de 2013 por presentar crisis convulsiva de aparición tardía con posible asociación con NCC y de éstos en el 57,1\%(4/7) fueron diagnosticados seropositivos según la prueba y mediante valoración por imagenología por RM o TAC, se observaron estructuras compatibles de ser estadios vesiculares o calcificaciones del metacéstodo de T. solium.; confirmándoseles NCC, (Evaluación neurológica no realizada, ni financiada por la investigación), cabe resaltar que tres de estos pacientes también presentan cuadro de cefalea frecuente. El cálculo de $\mathrm{p} \leq 0,05$ para el síntoma crisis convulsiva de aparición tardía, arrojo valor con significancia estadística en el estudio.

Accidentes cerebrovasculares y Alteración de conciencia

El $1,3 \%(2 / 159)$ y el $0,6 \%(1 / 159)$ de los participantes, manifestaron estar o haber sufrido algún tipo de acci- dente cerebrovascular o de alteración de conciencia; hallándose significancia estadística entre estas variables y la valoración serológica con valor de $\mathrm{p} \leq 0,05$.

Asociación de sintomatología y patología de mayor frecuencia

La asociación de sintomatología y patología en los diagnosticados seropositivos, se discriminó de la siguiente manera: cefalea y crisis convulsiva de aparición tardía 55,6\%(15/27); cefalea y accidente cerebrovascular $48,1 \%(13 / 27)$; cefalea y disminución de capacidad visual $44.4 \%(12 / 27)$; cefalea y alteración de conciencia $44,4 \%(12 / 27)$.

\section{Múltiple sintomatología}

EL 22,2\% (6/27) de los diagnosticados como seropositivos, manifestaron presentar o haber presentado simultáneamente en algún momento, sintomatología múltiple como: cefalea, crisis convulsiva, disminución de capacidad visual, accidentes cerebrovasculares y alteración de la conciencia entre otros, todos ellos de posible asociación a NCC. En la Gráfica 1se presenta los valores porcentuales de la sintomatología asociada a NCC, según el estudio.

\section{Valoración coproparasitológica}

A las 159 personas participantes, adicionalmente se les realizó un examen coproparasitológico. De la totalidad de la población muestreada, el 43.3\% (69/159) fueron positivas por presentar al menos un agente parasitológico gastrointestinal. Entre las entidades halladas, es de anotar que el 2\% (3/159) correspondió a la presencia de huevos de Taenia spp.; aún más relevante que entre los hallazgos, uno de ellos está incluido, en el grupo de las 27 personas diagnosticadas seropositivas y con presentación de sintomatología (crisis convulsiva de aparición tardía y cefalea) asociable a NCC, con confirmación posteriormente por TAC, de observación de estructuras calcificadas con localización parenquimal (Evaluación neurológica no realizada, ni financiada por la investigación) lo que permite sugerir, que dicha persona presenta o presentó una parasitosis mixta, tanto por el estadio adulto como el larval.

Cabe resaltar que de las personas seropositivas diagnosticadas en el estudio y que además presentaron sintomatología compatible con NCC, solo fue posible 
que a cuatro de ellas se les practicara valoración de imagenología por TAC o RM, debido al costo de estas técnicas; lo que hace pensar que es posible, que el número de casos de NCC en la población estudiada puedan ser mayor a los hallados. Las tres personas que por examen coproparasitológico se les identificó huevos de Taenia spp.; se informó de este resultado a cada uno de ellos como a los servicios de salud, para que se les suministrara el tratamiento antiparasitario, con el respectivo control y seguimiento médico.

\section{Discusión}

La cisticercosis humana, es una enfermedad con importancia médico-social y con relevancia económica, cuando su principal localización es el SNC como ocurre en América Latina (12), entre los síntomas asociados a NCC se identifica cefalea, crisis convulsivas de aparición tardía (del 40 al 60 \% de los casos), hipertensión, trastorno mental, visión borrosa $(13,14)$.

El 17\%(27/159) de seropositivos hallados en el estudio, el 67\% (18/27) manifestaron presentar como mínimo uno de los síntomas anteriormente enunciados, corroborando lo manifestado por varios autores, que un porcentaje significativo de las personas pueden cursar una NCC asintomática. El estudio realizado en México en el 2003; evidencia que la frecuencia de NCC es de $8,6 \%$ en seropositivos hospitalizados y señala que el $57 \%$ de los casos eran pacientes con alguna sintomatología asociada a NCC o con sospecha de la misma, el 30,6\% de estos, manifiestan tener una condición clínica regular. El estudio más reciente realizado en Colombia en 23 departamentos, revela una seroprevalencia general de cisticercosis del 8,5\%, como promedio nacional, durante el periodo comprendido entre los años 2008-2010, donde estimó específicamente para el departamento del Tolima un valor de seroprevalencia del 5,6\%; pero es de aclarar que el municipio de Coyaima no fue incluido en dicho estudio (1).

La investigación llevada a cabo en la ciudad de Popayán durante el periodo 2009-2010; registra una seroprevalencia del $35,87 \%$ en pacientes que asistieron a consulta médica, el $63 \%$ de ellos presentó al menos un síntoma asociable a NCC como con los evaluados en la presente investigación (15).

La cefalea fue la manifestación clínica de mayor frecuencia en las personas diagnosticadas seropositivas, aspecto en común con otros estudios de seroprevalencia como lo reportan Franco y Flórez $(15,1)$ donde éste, fue el síntoma común en los seropositivos, con $56,05 \%$ y $50,62 \%$ respectivamente;

En tanto que el trastorno mental sólo se presentó en el $1 \%$ de los seropositivos, valor similar a lo reportado por otros autores, lo que comprueba el hecho de que éste síntoma no es relevante en la asociación con la NCC (15).

El aspecto sociodemográfico sobresaliente en varios estudios es que el género femenino es de mayor seroprevalencia para esta parasitosis que el masculino, siendo en la presente investigación del 59,2\%. De las cuatro personas a quienes se les confirmó una NCC, tres de ellas son del género femenino y una del masculino; concordando esto en qué el género femenino es considerado, como un factor de riesgo inminente para adquirir la infección $\mathrm{RP}=1,60$ y $\mathrm{p} \leq$ 0,05 (1); esta situación no solo se presenta a nivel de Colombia sino también en países como Brasil, en investigación realizada en Rio de Janeiro el 62,5\% de las mujeres fueron confirmadas como positivas, Otro estudio realizado en Perú, reporta un hallazgo similar y argumenta qué esta condición puede obedecer a que las mujeres; se encargan de la manutención de los animales y a su vez son amas de casa, por lo tanto encargadas de la preparación de los alimentos por lo que el riesgo de contaminarse es mayor en relación al género masculino, coincidiendo esta situación con la población femenina evaluada en el municipio de Coyaima (16).

La presencia del ciclo epidemiológico de teniasis/ cisticercosis en una comunidad evidencia el poco desarrollo sociocultural y la deficiencia en infraestructura higiénicosanitaria y el bajo nivel educativo (17). Algunos autores destacan en sus investigaciones como factores de riesgo preponderantes asociados a la infección por teniasis/ cisticercosis, el libre acceso de los cerdos a las heces humanas y la contaminación del agua potable, como lo reportado para la comunidad de La yuca, Venezuela en el estado Yaracuy, donde se evidencia que el 93\% de la población realiza fecalismo al aire libre y no tienen acceso a agua potable (18),caso similar se observó en el presente estudio donde el $91.2 \%$ de la población del área urbana y periurbana del municipio de Coyaima, no cuenta con sistema de acueducto ni alcantarillado, presentándose una asociación estadísticamente significativa para esta condición, con valor de $p \leq 0,05$. La variable acueducto y alcantarillado es resaltada como factor de riesgo para la adquisición de la parasitosis causada por el binomio 
teniasis/cisticercosis, en las investigaciones de otros autores (15).

La tenencia de cerdos en la comunidad, es una de las variables relevantes halladas en el presente estudio, al igual que el fecalismo al aire libre o la inadecuada eliminación de excretas, facilitando el contacto de los porcinos con excremento humano, propiciando la infección de estos con el estadio vesicular de T. solium.;(19).

La NCC es una problemática evidente en Colombia, país endémico para esta parasitosis, donde existen zonas con condiciones favorables para la supervivencia del parásito y adicionalmente proclives a factores de riesgo asociadas a la misma (1). Otros países también presentan estadísticas abrumadoras tales como Chile, que considera a la NCC una enfermedad desatendida, por lo que el mismo autor concluye "la NCC es un problema vigente, con perfil de enfermedad desatendida por la ausencia de políticas específicas y transversales para su control y ni siquiera es de notificación obligatoria" (20). Brasil es un país que en 1983 consideró a la región de Riberao Preto una zona endémica de cisticercosis; para Rio de Janeiro el caso respecto a este padecimiento no fue diferente con 72 casos de esta parasitosis, con mayor frecuencia en mujeres con un $62,5 \%(21,22)$.

México reveló entre el periodo de 1943 y 1973 en necropsias un $2 \%$ para casos de NCC, razón por la cual fue incluido este hallazgo como problema de salud pública; se llegó a tener para 1995, 1600 casos de NCC, sin embargo tanto el complejo teniosis/ cisticercosis y la prevalencia de NCC en este país han decrecido considerablemente, probablemente por el establecimiento de un programa Nacional de Control de Taenia solium.; y la mejora en general de las condiciones de vida en México $(23,24)$.

Colombia no cuenta con políticas ni programas estatales de vigilancia y control para esta parasitosis, obteniéndose sólo indicios por los resultados epidemiológicos que al respecto se han evidenciado en las investigaciones reportadas por algunos autores en los últimos años (1).

\section{Conclusiones}

La NCC es una problemática evidente en el municipio de Coyaima, Departamento del Tolima donde se comprobó la coexistencia de un conjunto de variables asociadas a la presencia de esta parasitosis, como el hallazgo de personas teniásicas en la comunidad, por ser este el único requisito necesario para el establecimiento del binomio teniasis/cisticercosis en una región. Se estima que por cada persona teniásica puede haber ocho individuos cistercosos con o sin sintomatología manifiesta, de acuerdo a la dinámica de esta afección a nivel mundial.

En la población evaluada en el Municipio de Coyaima se confirmaron cuatro casos de NCC; cumpliéndose con cuatro criterios básicos: epidemiológico, sintomatológico, serológico y confirmación por imagenología (TAC y/o MR) (8).

Las dos técnicas de recolección serológica, implementadas en el presente estudio, siendo una de ellas la impregnación de círculos de papel filtro con sangre total y el empleo de la prueba de inmunodiagnóstico ELISA con: sensibilidad del $99 \%$, especificidad del $96.6 \%$, valor predictivo positivo y negativo del $100 \%$ y $97 \%$ respectivamente, permite inferir que la cantidad de muestra recolectada en el papel de filtro es suficiente para la valoración serológica con la técnica empleada. También cabe destacar que los valores de absorbancia de las muestras seropositivas, obtenidos en la prueba tanto para los sueros, como los discos impregnados con sangre total fueron similares e incluso en algunos casos superiores a los controles positivos incluidos en ésta. Los valores de absorbancia obtenidos en el ELISA para ambos casos, sueros y sangre total, fueron pareados para confrontar los resultados, hallándose concordancia en el ciento por ciento en éstos, en el diagnóstico tanto de las muestras positivas como negativas.

Para el desarrollo del ciclo epidemiológico es indispensable la presencia de estructuras resistentes a cambios ambientales, como son los huevos de $T$. solium.; para reproducir el estadio larval en el cerdo o el hombre, éste puede darse por la contaminación de aguas cercanas, las cuales son el abasto para el consumo humano como el de los porcinos. Las 27 personas halladas seropositivas en la investigación, 20 de ellas manifestaron hacer uso de agua sin ningún tipo de tratamiento como cloración, filtración o hervido, y al realizar el análisis de ésta variable, se identificó significancia estadística de la ji cuadrado con un valor de $\mathrm{p} \leq 0,05$ asociándole como posible causa para la adquisición de NCC en la población habitante del área urbana y periurbana del municipio de Coyaima Tolima.

El consumo de alimentos contaminados con huevos conlleva al curso de una cisticercosis y en el caso más grave 
a una NCC, finalmente el ser humano es un hospedero intermediario accidental, esta patología puede afectar musculatura a nivel general o el SNC e incluso a nivel ocular, lugares por los cuales el parásito tiene tropismo particular (4).

La crianza de cerdos en la población de Coyaima es una actividad ligada a la economía y gastronomía de esta región, la cual se realiza de manera no tecnificada y carente de control por parte de las autoridades sanitarias, el grupo de las 27 personas halladas seropositivas manifestó tener o haber tenido contacto directo con porcinos por práctica de crianza.

Entre los síntomas asociados a NCC se halló la cefalea, siendo uno de los más comunes en la población seropositiva, seguido de la crisis convulsiva de aparición tardía, el padecimiento por el cual acudieron a consulta médica siete personas y valoradas como seropositivas el $57,1 \%(4 / 7)$ a quienes se les confirmó NCC. Estos dos síntomas son los más destacados en varias referencias bibliográficas, debido a la atracción que presenta el cisticerco por la invasión del SNC humano y la presión ejercida por las estructuras calcificadas alojadas en el cerebro (25). Otras manifestaciones clínicas son la hipertensión, la pérdida de capacidad visual y alteración de la conciencia, ésta última aunque es un síntoma afín con la NCC, se considera como una condición posible, pero que en el presente estudio fue de poca relevancia en la población seropositiva.

El 67\%(18/27) de los seropositivos, presentaron alguna manifestación de posible asociación con NCC y el $33 \%(9 / 27)$ ausencia de estas, porcentaje que corrobora, qué algunos casos pueden cursar una NCC totalmente asintomática o como también presentarse una sintomatología exacerbada y baja presencia o total carencia de títulos de anticuerpos en suero o líquido cefalorraquídeo (LCR), además de la ausencia la respuesta inmunológica del hospedero y la no identificación de parámetros establecidos en el desarrollo de la parasitosis, dificultando aún más el diagnóstico de la enfermedad (1), a esto cabe agregar el hecho de que muchos casos deben ser analizados particularmente debido a las múltiples variantes sintomatológicas, debido principalmente a la ubicación de los estadios vesiculares o las calcificaciones en el SNC, como también se puede presentar en una NCC causada por cisticercos racemosos (25).

El presente estudio seroepidemiológico resalta la coherencia con varios autores respecto a las precarias condi- ciones higiénicas sanitarias que promueven el desarrollo del ciclo epidemiológico dando lugar a la cisticercosis humana y con especial énfasis a enfermedades como la NCC en ciertas comunidades. Es de anotar que en Colombia, se hace indispensable la creación de un programa de vigilancia para lograr no solo reducir los niveles de esta patología sino la erradicación de la misma, ejemplo de este tipo de programas se puede apreciar en el estado de Guerrero en México cuyas autoridades implementaron programas de educación en la comunidad y promovieron la vacunación de cerdos con péptidos recombinantes (potencialmente denominados como candidatos vacunales), logrando la disminución de la casuística para esta parasitosis(26).

\section{Agradecimientos}

Al grupo de investigación en Parasitología y Microbiología Tropical-GIMPAT, de la Universidad INCCA de Colombia.

Al laboratorio de Investigación en Parasitología de la Universidad INCCA de Colombia, Programa Profesional de Biología.

Al Centro de Estudios en Microbiología y Parasitología-CEMPA de la Facultad de Ciencias de la Salud Programa de Medicina de la Universidad del Cauca.

A la Liga Contra la Epilepsia Capítulo del Cauca-LCEC.

A la comunidad del Municipio de Coyaima por su participación en el proyecto.

Al cuerpo médico del Hospital San Roque del Municipio de Coyaima.

\section{Conflicto de intereses}

Los investigadores manifestamos no presentar conflicto de interés, todos los participantes en el estudio fueron debidamente informados, las muestras biológicas y la información que este contiene, se manejó con estricta reserva y tratamiento bioético.

\section{Financiación}

Este estudio fue financiado por la Universidad INCCA de Colombia aprobado en la convocatoria inter- 
na 007/02-2010/2011 y desarrollado por el Grupo de investigación de Parasitología y Microbiología tropical-GIPAMT de la Universidad INCCA de Colombia y el Centro de Estudios en Microbiología y Parasito-
logía-CEMPA de la Facultad de Ciencias de la Salud Programa de Medicina de la Universidad del Cauca y la colaboración de la Liga Contra la Epilepsia Capítulo del Cauca-LCEC.

\section{ANEXOS}

Tabla 1. Demografía de la población muestreada ( $n=159)$ y valor porcentual de personas seropositivas y variables asociadas a NCC, en la población del área urbana y periurbana del municipio de Coyaima-Tolima

\begin{tabular}{|c|c|c|c|}
\hline & $\%$ & Seropositivos & $\%$ IC $95 \%$ \\
\hline \multicolumn{4}{|l|}{ Género } \\
\hline Femenino & 68 & 16 & 59,2 \\
\hline Masculino & 32 & 11 & 40,7 \\
\hline \multicolumn{4}{|l|}{ Grupos Etáreos } \\
\hline 1-23 años & 18,2 & 7 & 26 \\
\hline 24-35 años & 32,1 & 8 & 29,6 \\
\hline Mayores a 36años & 48 & 12 & 44,4 \\
\hline \multicolumn{4}{|l|}{ Procedencia } \\
\hline Periurbana & 34 & 9 & 33,3 \\
\hline Urbana & 66 & 18 & 66,6 \\
\hline \multicolumn{4}{|l|}{ Área de Ocupación } \\
\hline Desempleado & 44,6 & 8 & 29,6 \\
\hline Empleado & 8,8 & 1 & 3,7 \\
\hline Independiente & 28,3 & 9 & 33,3 \\
\hline Estudiante & 17,6 & 8 & 29,6 \\
\hline Pensionado & 0,63 & 1 & 3,7 \\
\hline \multicolumn{4}{|l|}{ Régimen de Salud } \\
\hline ARS & 1,2 & 0 & 0 \\
\hline EPS & 83,6 & 26 & 96,3 \\
\hline SISBEN & 13,2 & 1 & 3,7 \\
\hline Desplazado & 0,63 & 0 & 0 \\
\hline Ninguno & 1,2 & 0 & 0 \\
\hline \multicolumn{4}{|l|}{ Nivel Educativo } \\
\hline Sin escolaridad & 12 & 2 & 7,4 \\
\hline Primaria & 46,5 & 14 & 51,8 \\
\hline Secundaria & 33,3 & 8 & 29,6 \\
\hline Tecnológica & 6,3 & 3 & 11,1 \\
\hline Universitario & 2 & 0 & 0 \\
\hline
\end{tabular}


Tabla 2. Valor porcentual de seroprevalencia de anticuerpos anticisticerco con posible asociación a variables higiénico sanitarias, procedencia y tenencia de cerdos en la población del área urbana y periurbana del municipio de Coyaima-Tolima

\begin{tabular}{|lccc|}
\hline \multicolumn{1}{|c}{ Variable } & SEROPOSITIVOS & P IC 95\% \\
& $\mathrm{n} /$ total & $\%$ & \\
\hline Tenencia de porcinos & $7 / 37$ & 18,9 & 0,72 \\
$\mathrm{Si}$ & $20 / 122$ & 16,4 & \\
$\mathrm{No}$ & & & 0,000 \\
\hline Fuentes de agua & 0 & 0 & \\
Tratadas & $27 / 145$ & 18,6 & 0,000 \\
Sin tratamiento & & & \\
\hline Procedencia & $18 / 105$ & 17,4 & \\
Urbana & $9 / 54$ & 16.6 & \\
Periurbana & & & \\
\hline
\end{tabular}

Tabla 3. Valor porcentual de seroprevalencia de anticuerpos anticisticerco y asociación a sintomatología compatible con NCC en la población del área urbana y periurbana del municipio de Coyaima-Tolima

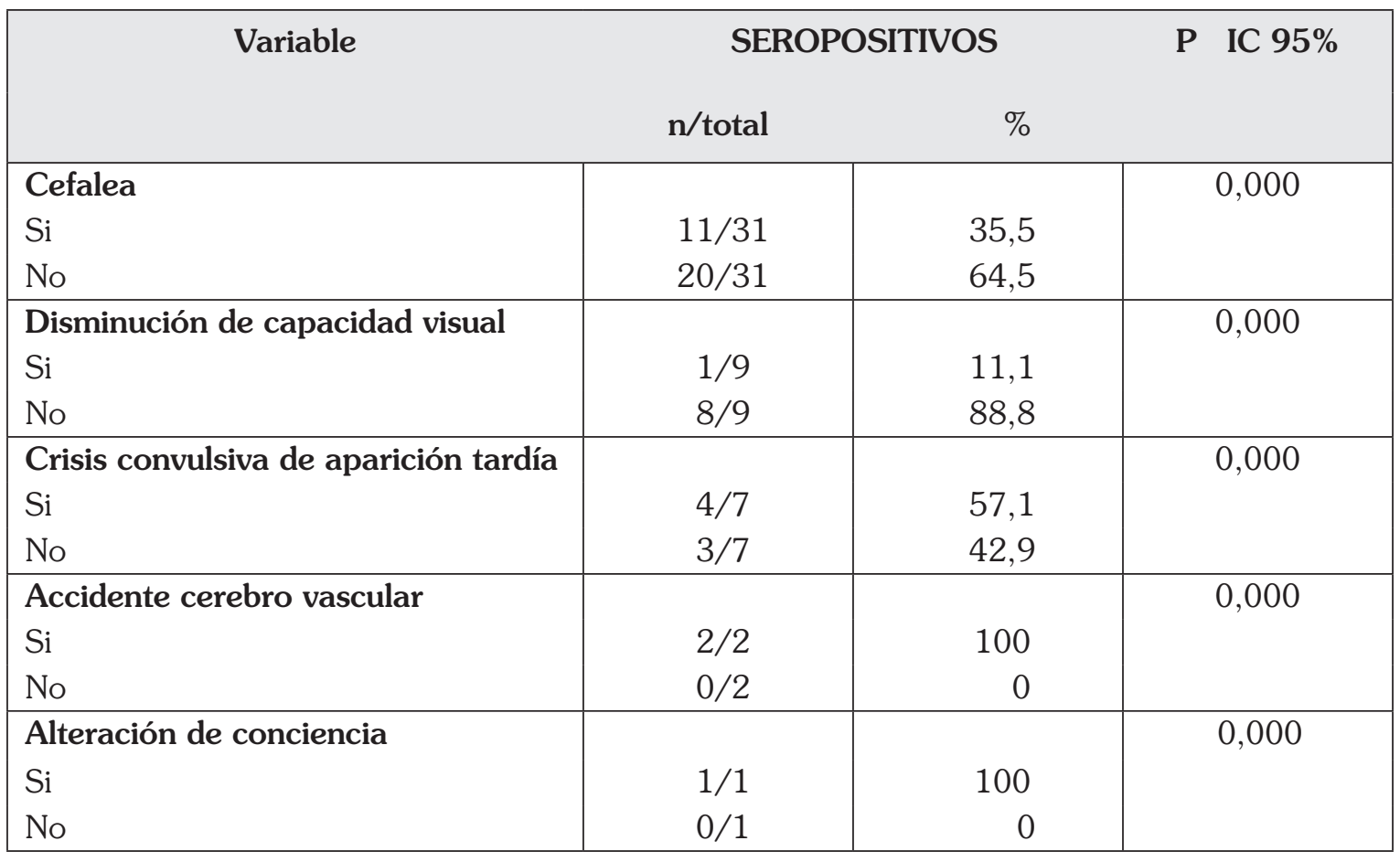




\section{Valores porcentuales de sintomatología asociados a Neurocisticercosis-NCC}

$\square$ Cefálea
$\square$ Crisis convulsiva de aparición tardia
$\square$ Alteración de conciencia
$\square$ Cefálea y accidente cerebrovascular
$\square$ Cefálea y alteración de conciencia

口Disminución de capacidad visual

- Accidentes cerebrovasculares

$\square$ Cefálea y crisis convulsiva de aparición tardía

口Cefálea y disminución de capacidad visual

Múltiple sontomatología

55,6

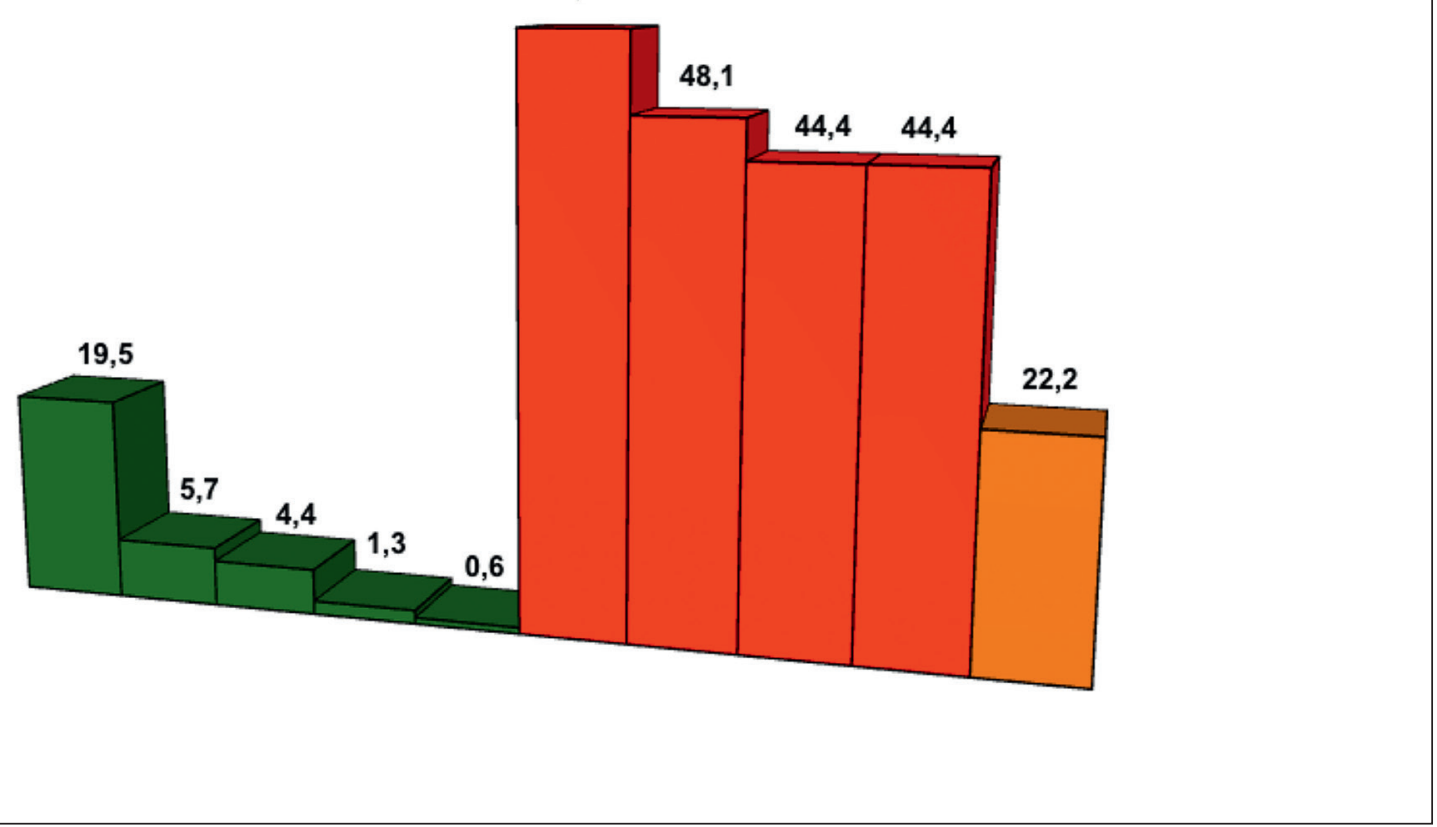

\section{Referencias}

1. Flórez A, PA, Salamanca L, Pastrán S, Vargas N, Beltrán M., Enríquez Y, Peña A, Villareal, Rincón C, Garzón I, Muños L, Guasmayan L, Valencia C, Parra S. Cisticercosis en Colombia. Estudio de seroprevalencia 2008-2010. Acta Neurológica Colombia. 2012; 29(2):73-85.

Disponible en: http://www.scielo.org.co/pdf/anco/ v29n2/v29n2a03

2. Nogales J, Arriagada C, Salinas R. Tratamiento de la Neurocisticercosis: Revisión crítica. Rev Méd. Chile. 2006; 134:789-796. Disponible en: http://www.scielo.cl/pdf/ rmc/v134n6/art17.pdf

3. Flisser, A. Cisticercosis: enfermedad desatendida. Bol Med

Hosp Infant Mex. 2011; 68(2):138-145.

Disponible en: http://www.scielo.org.mx/scielo.php?script=sci_arttext\&pid=S1665-11462011000200010

4. Vásquez L, Giraldo J, Agudelo P, Campo V, Vergara D. Experiencia para el control de la cisticercosis en el departamento del Cauca. Biomédica. 2011; 31 (3):300-315. Disponible en: http://www.revistabiomedica.org/index. php/biomedica/article/viewFile/536/665

5. Flisser A, Sarti E, Lightowlers M, Schantz P. Neurocysticercosis, regional status, epidemiology, impact and control measures in the Americas. Acta Trop. 2003; 87:43-51. Disponible en: http://www.ncbi.nlm.nih.gov/ pubmed/12781377 
6. Naquira C. Las zoonosis parasitarias: Problema de salud pública en el Perú. Revista peruana de Medicina Experimental y Salud Publica. 2010; 27(4):494-497. Disponible en: http://www.redalyc.org/articulo.oaid=36318511001

7. U.S. Departament of healt and human services. Recommendations of the international Task Force for Disease Eradication. 1993; 42(RR-16).

Disponible en: http://www.cdc.gov/mmwr/PDF/rr/ rr4216.pdf

8. Walteros D. Cisticercosis: situación de parasitosis. Informe Final de Evento Cisticercosis. 2009.

Disponible en: http://www.ins.gov.co/lineas-de-accion/ Subdireccion-Vigilancia/Informe\%20de\%20Evento\%20 Epidemiolgico/Cisticercosis2009.pdf

9. Giraldo J, Piraguata M, Bachiller Neiffe M. Estandarización de una prueba inmunodiagnóstica ELISA para la detección de anticuerpos humanos contra el metacéstodo de Taenia solium.; en extracto crudo y fracción proteíca de 53 kDa. Biomédica. 2002; 22(s1):63.

10. Andriamandimby J, Heraud L, Randrianasolo T, Rafisandratantsoa S, Andriama- Monjy V, Reichard. Dried-blood spots: a cost- effective field method for the detection of chikungunya virus circulation in remote areas. Internacional Journal for Parasitology: Parasites and Wildlife. 2013;3:15-19. Disponible en:http://journals.plos.org/ plosntds/article?id=10.1371/journal.pntd.0002339

11. Thangavelu A, Dhinakar R, Elankumaran S, Koteeswaran A. Evaluation of a filter paper blood sampling technique for quantitative assessment of antibodies to infectious bursal disease virus. Tropical Health and Production. 2000; 32:179-182. Disponible en: http://link.springer.com/article/10.1023\%2FA\%3A1005239816285?LI=true\#page-2

12. Flisser A, Plancarte A, Avila G. Application of diagnostic methods for cysticercosis and taeniosis to epidemiological studies. In: Taenia solium: Taeniasis/cysticercosis. Ed. Universo, Lima, Perú. 1999: 39-52.

13. Mahanty S, García HH. Cysticercosis Working Group in Perú. Cysticercosis and neurocysticercosis as pathogens affecting the nervous system. Prog. Neurobiol. 2010; 91:172-184.Disponible en: http://www.sciencedirect. com/science/article/pii/S0301008209002020

14. Montero J, Rojas R. Estudio retrospectivo de la seroprevalencia de Neurocisticercosis en Colombia dentro del periodo de enero de 1995 a diciembre de 2005. Trabajo de grado bacteriología. Bogotá: Pontificia Universidad javeriana. Facultad de ciencias. Departamento de bacteriología. 2006:110-118.

Disponible en: http://www.javeriana.edu.co/biblos/tesis/ ciencias/tesis170.pdf

15. Franco C, Giraldo J, Vásquez R. Detección de anticuerpos anticisticerco en pacientes que asistieron a consulta médica durante el periodo 2009 a la Liga Contra la Epilepsia. Capitulo Cauca, Revista UNINCCA. 2012; 18(1):79-93. Disponible en: https://www.unincca.edu.co/images/stories/pfd/revista-cientifica-v18-1.pdf
16. Ayala ER, Medina SA, Morales JA. Cisticercosis humana y sus asociación con factores epidemiológicos y clínicos en los Andes centro y sur de Perú. Revista epidemiológica del Perú. 2014; 18(1):1-6 Disponible en: http://www. redalyc.org/pdf/2031/203131355005.pdf

17. Sotelo J. Controversias y perspectivas en Neurocisticercosis. En: Cisticercosis: Guía para profesionales de la salud. Ed fondo de Cultura económica, México. 2006;capVIII: 238-244. Disponible en: http://www-lab.biomedicas. unam.mx/cistimex/s1/Cap8.pdf

18. Cortez M, Aguilar C, Rojas G, Boggio G., Lares M, Ferrer E, Parkhouse M. Evidencia de transmisión activa de cisticercosis por Taenia solium en comunidades rurales de Venezuela. Revista Biomédica. 2011; 31(s3):300-315.

Disponible en: http://www.revistabiomedica.org/index. php/biomedica/article/viewFile/543/672

19. Agudelo S, Gómez L, Coronado X, Orozco A, Valencia C, Restrepo L, Galvis L, Palacio L. Prevalencia de parásitos intestinales y factores asociados en un corregimiento de la Costa Atlántica Colombiana. Revista de salud pública. 2008; 10 (4): 633-642. Disponible en: http:// www.scielosp.org/scielo.php?script =sci_arttext\&pi$\mathrm{d}=$ S0124-00642008000400013

20. Fica A, Castro M, Sotoa, Flores C, Oelker C, Weitzel T. Neurocisticercosis - una enfermedad desatendida en Chile. Revista Chil Infect. 2012;29(1): 72- 81. Disponible en: http://scielo-test.scielo.cl/pdf/rci/v29n1/art12.pdf

21. Takayanagui OM, Jardim E. Aspectos clínicos da neurocisticercose: analise de 500 casos. Arq Neuropsiquiatr. 1983; 41:50-63. Disponible en: http://www.scielo.br/pdf/anp/ v41n1/04.pdf

22. Sato M. Cisticercosis, un obstáculo para el desarrollo: la situación en Brasil. Biomédica. 2011; 31(3):300 -315. Disponible en: http://www.revistabiomedica.org/index. php/biomedica/article/viewFile/543/672

23. Mendes E, Santos da Silva S \& La Terza Fonseca E, Regina Ribeiro de Souza Raimundo, Wilson de Carvalho. A neurocisticercose humana na Baixada Fluminense, Estado de Rio de Janeiro, Brasil. Arq. Neuro-Psiquiatr. 2005; 63(4). Disponible en: http://www.scielo.br/scielo.php?script=sci_arttext\&pid=S0004-282X2005000600027

24. Flisser A. Control de la teniasis y la cisticercosis en México, Biomédica. 2011; 31(s3): 300-315. Disponible en: http://www.revistabiomedica.org/index.php/biomedica/ article/viewFile/536/665

25. Del Brutto OH. Neurocisticercosis: actualización en diagnóstico y tratamiento. Rev. Neurología. 2005;20(8): 412-18. Disponible en: http://clinicalevidence.pbworks. com/w/file/fetch/77449862/Neurocisticercosis. Diagnóstico y tratamiento.pdf.

26. Aluja A, Suarez R, Sciutto E, Biom I, Morales J, Veter $\mathrm{D}$, Martínez J, Villalobos N. Evaluación del impacto de un programa de control de teniasis-cisticercosis Rev. Salud publica México. 2014;56(3):259-265. Disponible en: http://www.scielo.org.mx/scielo.php?script=sci_arttext\&pid=S0036-36342014000300011 